\title{
Micro/Nano-structures Formation on ITO Thin Film Irradiated by Dif- ferent Femtosecond Laser Wavelengths
}

\author{
C.W. Cheng ${ }^{1 *}$, I.M. Lee ${ }^{2}$, W.C. Shen ${ }^{1}$, C.Y. Lin $^{1}$ and J.S. Chen ${ }^{3 *}$ \\ ${ }^{* 1}$ ITRI South, Industrial Technology Research Institute, No. 8, Gongyan Rd., Liujia Shiang, Tainan County 734, Taiwan, \\ R.O.C. \\ E-mail: CWCheng@itri.org.tw (C.W. Cheng) \\ ${ }^{2}$ Department of Mechanical Engineering,National Chung Cheng University, No. 168, University Rd., Min-Hsiung, Chia-Yi \\ 621, Taiwan, R.O.C. \\ ${ }^{* 3}$ Department of Mechanical Engineering, National Chung Hsing University, 250 Kuo Kuang Rd., Taichung 402, Taiwan \\ R.O.C. \\ E-mail: MichaelChen@dragon.nchu.edu.tw(J.S.Chen)
}

\begin{abstract}
This study presented the fabrication of micro/nano crystalline indium tin oxide (c-ITO) patterns on glass substrate using a femtosecond laser-induced crystallization followed by chemical etching. In the proposed approach, an amorphous ITO (a-ITO) thin film was transformed into a c-ITO micro/nano structure over a predetermined area using femtosecond laser beam irradiation, under two different wavelengths, $1064 \mathrm{~nm}$ and $532 \mathrm{~nm}$, of $1 \mathrm{MHz}$ repetition rate femtosecond fiber laser. The residual a-ITO thin film was removed by an etchant solution, in order to form a uniform c-ITO pattern. The formation was due to the heat accumulation effect provided by the high-repetition rate of the femtosecond laser pulses. When the laser power approached the ablation threshold of the a-ITO thin film, periodic-like c-ITO nanostructures with one-quarter of the irradiation laser wavelength were formed by linearly-polarized laser beam irradiation.

DOI:10.2961/jlmn.2010.03.0006
\end{abstract}

Keywords: femtosecond laser, ITO, crystalline, patterning, heat accumulation effect

\section{Introduction}

Indium-tin oxide (ITO) is a widely used as a transparent conducting material with high transparency in the visible region of the spectrum. The resistivity of an ITO pattern should be maintained as low as possible in order to obtain a high response for functional devices, such as flat-panel displays, solar cells, and touch panels. A crystalline structure usually has a lower resistivity than an amorphous structure [1]; therefore, innovation in the patterning of cITO technology has significant impact on fabrication cost and processing time.

The conventional fabrication of a c-ITO pattern is based on a photolithographic process, where an a-ITO thin film undergoes thermal annealing at a temperature higher than $200{ }^{\circ} \mathrm{C}$ [1]. It is a multiple process that involves photoresist coating and backing, photoresist exposure and developing, etching, and photoresist stripping. Thus, during the heat treatment, it is easy to damage other layers or the heat sensitive substrates. Laser ablation is a natural technology intended for use in patterning ITO film, and many researches have investigated the ablation process by nanosecond, picosecond, and femtosecond lasers [2-8]. However, the ablation process requires sufficient laser fluence to evaporate the materials. The elevated ridges of edges and ITO residues on an ablated bottom easily occur, which may cause shorting of the structure or the adjacent electrodes. To overcome the above disadvantages, [9] utilized a nanosecond excimer laser to pattern a-ITO thin films using the crystallization effect. However, the results showed that the thermal effects induced by the relatively long laser pulse degraded patterning precision. Moreover, the process re- quired the use of a patterning mask, and thus, the characteristic size of the resulting structure was limited by the optic diffraction properties of the mask.

This study proposes a novel maskless technique to fabricate crack-free c-ITO micro patterns and periodic-like cITO nanostructures from a-ITO thin film using femtosecond laser-induced crystallization, followed by chemical etching. Crystallization under different conditions, including laser wavelength, laser pulse energy, and scanning speed are studied. The irradiated ITO film is then etched in an oxalic acid solution to remove residual a-ITO regions, as a-ITO has a higher etching rate than c-ITO. The results show that, given an appropriate choice of laser parameters, crack-free c-ITO patterns and periodic-like c-ITO nanostructures can be accomplished.

\section{Experimental}

The experiments were performed using a fundamental and double-frequency high repetition rate femtosecond fiber laser (FemtoPower 1060-3 $\mu \mathrm{J}$-s, Fianium Inc.), with a pulse width of $<500 \mathrm{fs}$, a wavelength of $1064 \mathrm{~nm}$ and 532 $\mathrm{nm}$, a high-repetition rate of $1 \mathrm{MHz}$, and a maximum pulse energy of $\sim 3 \mu \mathrm{J}$ at $1064 \mathrm{~nm}$. The laser beam entered an objective lens (numerical aperture 0.26, M Plan Apo NIR, Mitutoyo), and was incident in the normal direction on the surface of an a-ITO coated specimen, which was mounted on an X-Y positioning stage. The focal spot diameter of the specimen surface was approximately $7 \mu \mathrm{m}$ and $3.5 \mu \mathrm{m}$ wavelengths, for $1064 \mathrm{~nm}$ and $532 \mathrm{~nm}$, respectively. The cITO structures were then fabricated by translating the sample stage, while controlled by a PC-based micro- 
positioning system, to obtain a precision better than $1 \mu \mathrm{m}$. In the experiments, a-ITO thin films with a thickness of $\sim 100 \mathrm{~nm}$ were deposited on glass substrates (NEG OA10) by a DC magnetron sputtering system.

After laser processing, the samples were immersed in a $0.05 \mathrm{~mol} / \mathrm{L}$ oxalic acid etchant at $50^{\circ} \mathrm{C}$ for $2 \mathrm{~min}$. Due to the rapid etching rate of the a-ITO thin film, which is greater than the c-ITO structure [9], the etching process resulted in the complete removal of the residual a-ITO film, leaving only the crystalline c-ITO pattern. The surface characteristics of the crystalline structures were then observed by scanning electron microscopy (SEM). The crystalline and optical properties were measured by X-ray diffraction method (XRD, Bruker Smart APEX CCD X-ray) and spectrophotometer, respectively.

\section{Results and Discussion}

Figures 1 and 2 present the SEM images of the c-ITO line patterns fabricated under laser wavelengths of $1064 \mathrm{~nm}$ and $532 \mathrm{~nm}$, and laser fluence in the range 278 2145 $\mathrm{mJ} / \mathrm{cm}^{2}$ and $364 \sim 1312 \mathrm{~mJ} / \mathrm{cm}^{2}$, respectively. The scanning speed of $0.25 \mathrm{~mm} / \mathrm{s}$ and laser beam were linearly polarized in a direction parallel to the scan direction. Due to the Gaussian property of the beam profile, the middle of each line was irradiated by laser energy that was greater than at the edges. In the c-ITO line patterns, as shown in Figs. 1(a) (d) and Figs. 2(a) (d), the peak energy intensity of the laser beam was higher than the crystallization threshold, but lower than the ablation threshold. Thus, c-ITO line patterns were formed on the surface of the glass substrate, and the a-ITO film was completely removed following the chemical etching process. In Figs. 1(e) (f) and Figs. 2(e) (f), the peak energy intensity of the laser beam was higher than the ablation threshold, and thus, the center of the line was ablated. However, the outer edges of the irradiated paths were subject to the lower energy input, and thus, the ablated channels were bordered by regions of the c-ITO line patterns.

In Figs. 1(a) (d) and Figs. 2(a) (d), the pattern widths were sensitive to the spatial intensity distribution of the focused laser beam. Assuming a Gaussian incident beam profile, pattern width $W$ is related to the irradiation laser fluence $F$ by:

$$
W^{2}=2 \omega_{e}^{2} \ln \left(F / F_{t h}\right)
$$

where, $F_{t h}$ is the multiple pulse crystallization threshold fluence, and $\omega_{e}$ is the effective beam radius at the interaction surface.

Figure 3 shows the variation of the c-ITO pattern width, with the laser wavelengths of $1064 \mathrm{~nm}$ and $532 \mathrm{~nm}$. The straight lines obtained by the curve fitting of the experimental data confirmed a logarithmic dependence between the pattern width and the laser fluence. Moreover, the crystallization threshold fluences were approximately $248 \mathrm{~mJ} / \mathrm{cm}^{2}$ and $253 \mathrm{~mJ} / \mathrm{cm}^{2}$ for the laser wavelengths of $1064 \mathrm{~nm}$ and $532 \mathrm{~nm}$, respectively.

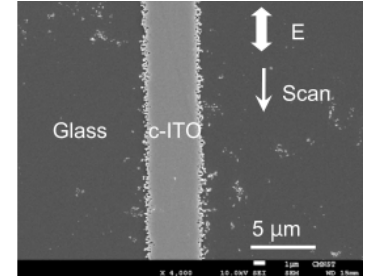

(a)

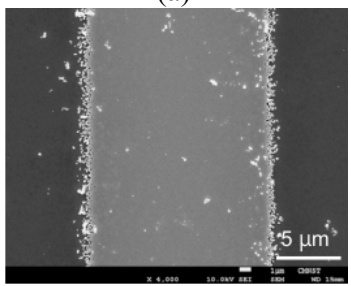

(c)

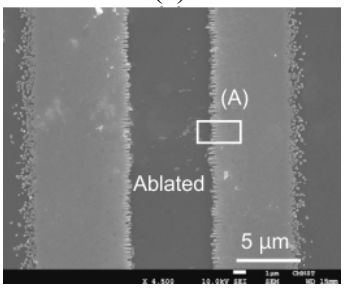

(e)

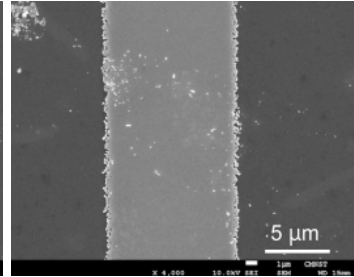

(b)

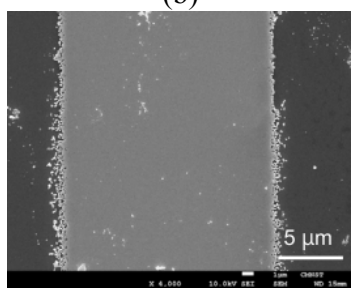

(d)

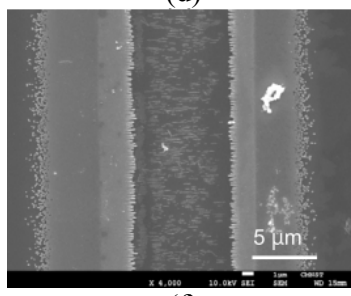

(f)
Fig. 1 SEM images of c-ITO line patterns, fabricated under a laser wavelength of $1064 \mathrm{~nm}$ and laser fluences of: (a) $278 \mathrm{~mJ} / \mathrm{cm}^{2}$, (b) $364 \mathrm{~mJ} / \mathrm{cm}^{2}$, (c) $614 \mathrm{~mJ} / \mathrm{cm}^{2}$, (d) $881 \mathrm{~mJ} / \mathrm{cm}^{2}$, (e) $1492 \mathrm{~mJ} / \mathrm{cm}^{2}$, and (f) $2145 \mathrm{~mJ} / \mathrm{cm}^{2}$. The scanning speed was $0.25 \mathrm{~mm} / \mathrm{s}$ in all cases.

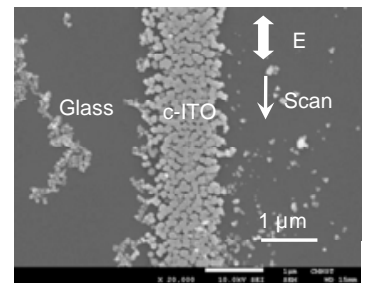

(a)

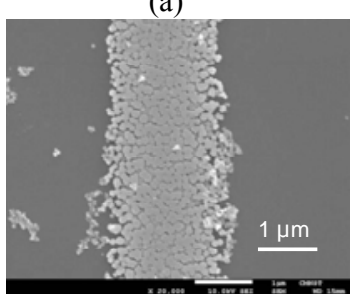

(c)

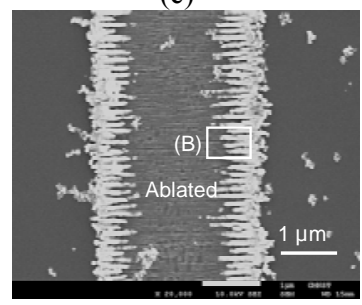

(e)

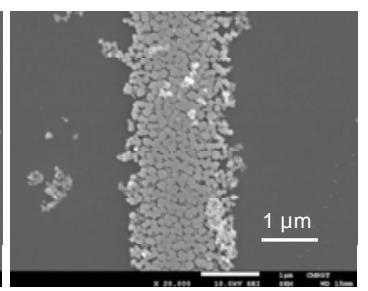

(b)

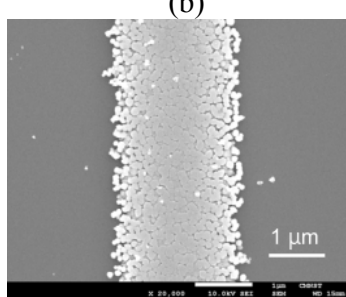

(d)

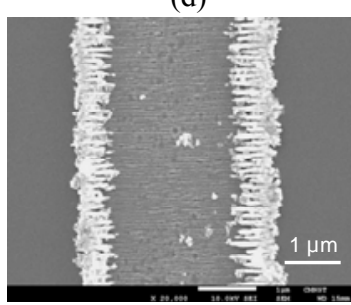

(f)
Fig. 2 SEM images of c-ITO line patterns fabricated under a laser wavelength of $532 \mathrm{~nm}$ and a laser beam, linearly polarized in a direction parallel to the scan direction, and laser fluences of: (a) $364 \mathrm{~mJ} / \mathrm{cm}^{2}$, (b) $448 \mathrm{~mJ} / \mathrm{cm}^{2}$, (c) $528 \mathrm{~mJ} / \mathrm{cm}^{2}$, (d) $574 \mathrm{~mJ} / \mathrm{cm}^{2}$, (e) $872 \mathrm{~mJ} / \mathrm{cm}^{2}$, and (f) $1312 \mathrm{~mJ} / \mathrm{cm}^{2}$. The scanning speed was 0.25 $\mathrm{mm} / \mathrm{s}$ in all cases.

In Figs. 1(a) (d), the c-ITO lines were free of microcracks, and showed the characteristic of a smooth, unblemished surface. Thus, the high repetition rate femtosecond 
fiber laser (i.e. $1 \mathrm{MHz}$ ) resulted in a more uniform crystallization effect than that achieved by a low repetition rate laser [9]. Previous studies have shown that the processing of certain bulk transparent glasses, under a high repetition rate femtosecond laser (i.e. $>200 \mathrm{kHz}$ ), resulted in an accumulation of heat, which minimizes the thermal cycling effect between successive laser pulses, and suppresses micro-crack formation [10].

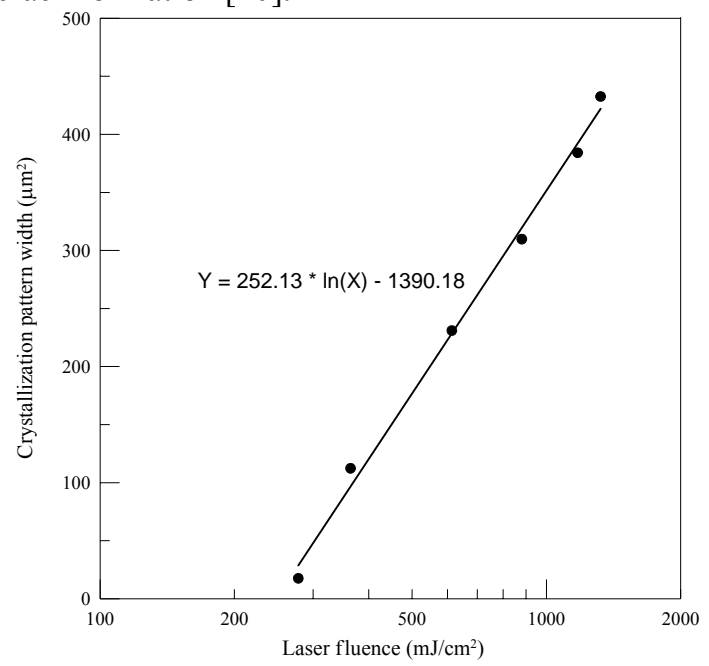

(a)

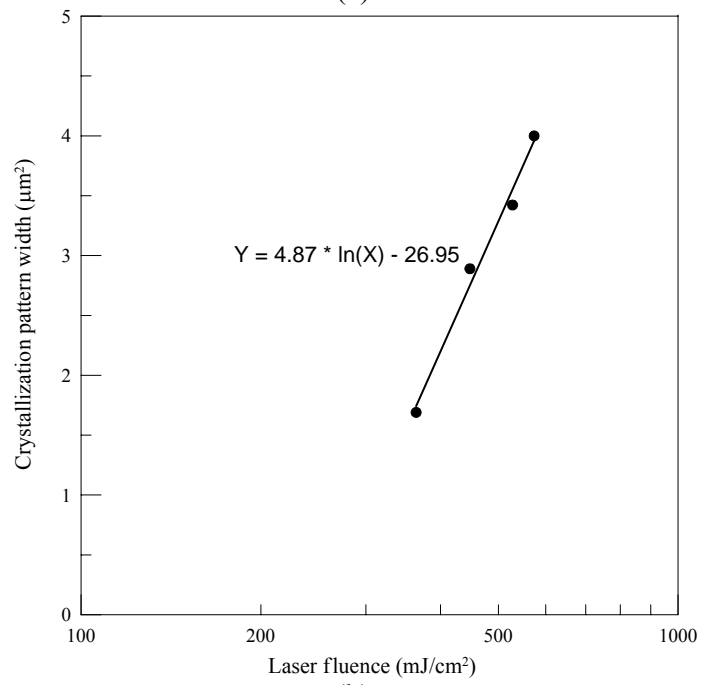

(b)

Fig. 3 Variations of pattern widths, with laser fluence under laser wavelengths of (a) $1064 \mathrm{~nm}$ and (b) $532 \mathrm{~nm}$.

As shown in Figs. 1 2, the heat accumulation effect was also present in the a-ITO thin film specimens, which were processed under the current high repetition rate (1 $\mathrm{MHz}$ ) femtosecond fiber laser. The effective cooling time, $t_{c}$ of the present a-ITO thin film was calculated at about 41 $\mu$ s and $10 \mu$ s of laser wavelengths of $1064 \mathrm{~nm}$ and $532 \mathrm{~nm}$, respectively, thus, $t_{c}=d_{v}{ }^{2} / D$ [11], where $d_{v}$ is the diameter of the focused laser beam spot $\sim 7 \mu \mathrm{m}$ for $1064 \mathrm{~nm}$, and $\sim 3.5$ $\mu \mathrm{m}$ for $532 \mathrm{~nm}$, and $D$ is the thermal diffusivity of a-ITO ( $\left.1.2 \times 10^{-6} \mathrm{~m}^{2} / \mathrm{s}\right)$. When the a-ITO was irradiated by a 1064 $\mathrm{nm}$ laser, the effective cooling time of $41 \mu \mathrm{s}$ was much higher than the time interval between successive pulses (1 $\mu$ s for a $1 \mathrm{MHz}$ repetition rate). Thus, an obvious heat accumulation effect occurred within the local region of the ITO film. However, when the a-ITO was irradiated by a $532 \mathrm{~nm}$ laser, the effective cooling time of $10 \mu$ s was only slightly higher than the time interval between successive pulses, resulting in a lower heat accumulation effect occurring within the local region of the ITO film. As a result, the surface quality of the line patterns shown in Figs. 2(a) (d) was poor.

Figure 4 shows the magnified image of area (A) of Fig. 1(e); and area (B) of Fig. 2(e), respectively. As seen, the periodic-like c-ITO nanostructures, under periods of $\sim 250$ $\mathrm{nm}$ and $\sim 116 \mathrm{~nm}$, were formed at laser wavelengths of $1064 \mathrm{~nm}$ and $532 \mathrm{~nm}$, respectively. The formation of the periodic-like nanostructures was attributed to the secondorder harmonic waves within the femtosecond laser beam [12-14]; $\lambda / 2 n$, where $\lambda$ is the wavelength of the incident laser, and $n$ is the refractive index of the irradiated material. Since the band gap in the a-ITO thin film (band gap $~ 3.6$ $\mathrm{eV}$ ) was much larger than the photon energy of $1064 \mathrm{~nm}$ $(1.16 \mathrm{eV})$ and $532 \mathrm{~nm}(2.33 \mathrm{eV})$, the incident laser created a dense electron plasma through multiphoton excitation at the surface of the irradiated material [15]. In this experiment, the magnitude $n$ of the ITO thin film for $1064 \mathrm{~nm}$ and $532 \mathrm{~nm}$ laser was $\sim 2$, under calculated periods of 266 $\mathrm{nm}$ and $133 \mathrm{~nm}$, respectively, which is close to the experimental data shown in Fig. 4. Figure 5 shows the SEM image of the c-ITO line patterns fabricated under a laser wavelength of $532 \mathrm{~nm}$, a scanning speed of $10 \mathrm{~mm} / \mathrm{s}$, and laser fluence of $572 \mathrm{~mJ} / \mathrm{cm}^{2}$. In addition, the period was about $\sim 116 \mathrm{~nm}$. The potential applications of the periodiclike c-ITO nanostructures included nanopatterned surfaces for micro-sensors and nanograting for optical devices [16].

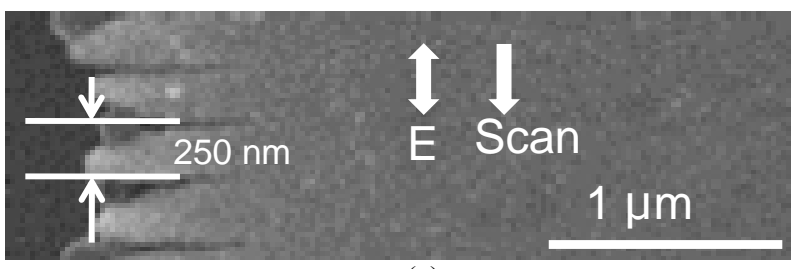

(a)

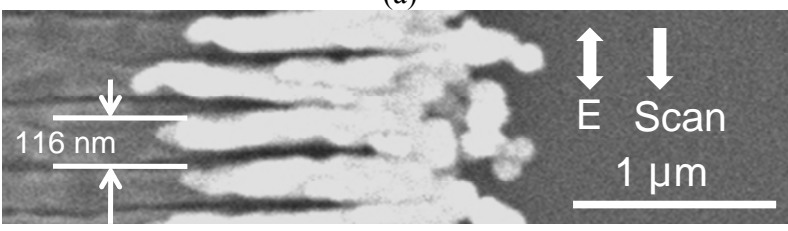

(b)

Fig. 4 The magnified image of (a) area (A) of Fig. 1(e); and (b) area (B) of Fig. 2(e).

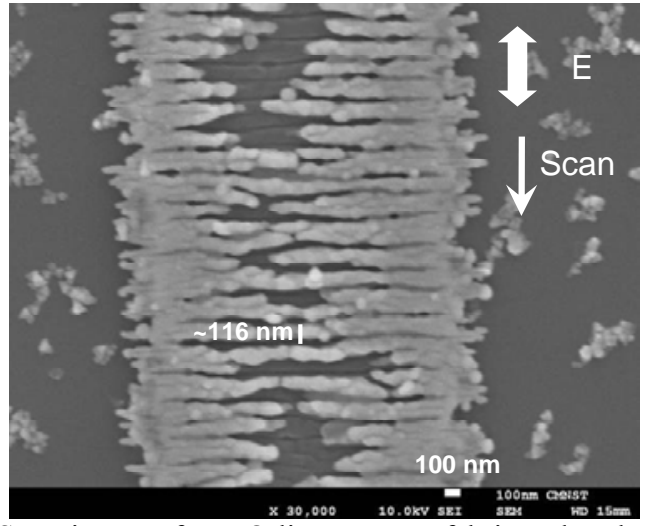

Fig. 5 SEM image of c-ITO line patterns fabricated under a laser wavelength of $532 \mathrm{~nm}$, a scanning speed of $10 \mathrm{~mm} / \mathrm{s}$, and laser fluence of $572 \mathrm{~mJ} / \mathrm{cm}^{2}$. 
In order to study the large area crystalline structure and optical transparency of the fabricated c-ITO structure, the analyzed sample was fabricated by scanning an area of $15 \times 10 \mathrm{~mm}^{2}$ with a laser wavelength of $1064 \mathrm{~nm}$, a scanning speed of $10 \mathrm{~mm} / \mathrm{s}$, and a laser fluence of $364 \mathrm{~mJ} / \mathrm{cm}^{2}$ (based on the results in Fig. 1 (b)). Figure 6 shows the XRD results for the as-posited and laser-crystallized ITO films. As seen in Fig. 6(a), a-ITO thin films did not show crystalline, and the diffusion hump at $2 \theta=24^{\circ}$ was due to the glass substrate. In Fig. 6(b), crystalline peaks of indium oxide at (222) and (440) indicated the formation of a crystalline structure after laser crystallization.

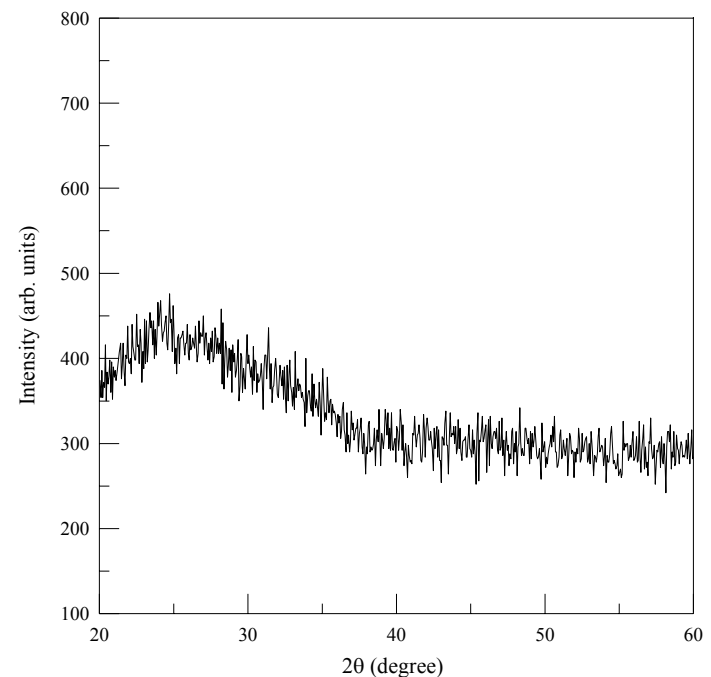

(a)

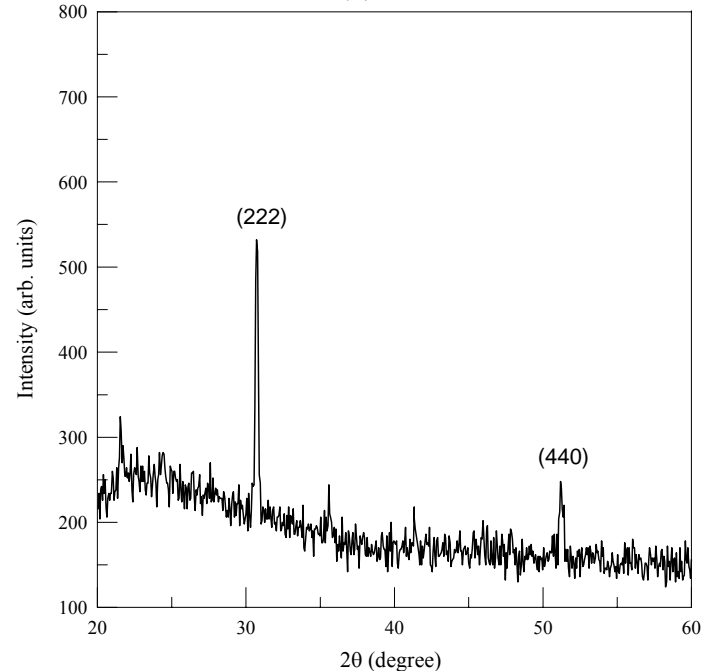

(b)

Fig. 6 X-ray diffraction (XRD) result of (a) as-deposited and (b) laser-crystallized ITO films under a laser wavelength of $1064 \mathrm{~nm}$

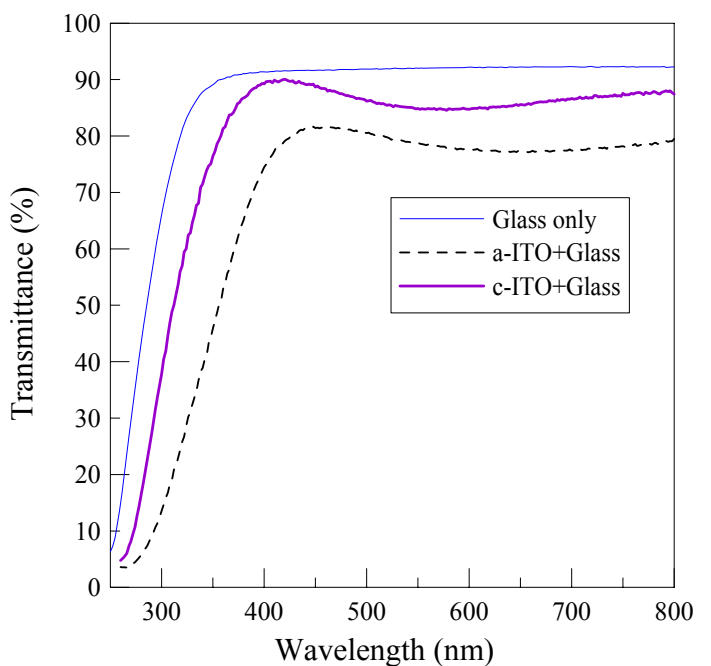

Fig. 7 Optical transmittance spectrum of the ITO film

Figure 7 shows the optical transmittance spectrum of the ITO film before and after femtosecond laser crystallization. As seen, the transparency in the visible range was increased from 78.8 to $84.9 \%$ at a wavelength of $550 \mathrm{~nm}$.

\section{Conclusions}

This study proposed a novel maskless procedure for the fabrication of micro/nano c-ITO structures on glass substrates. In the proposed approach, the a-ITO film was irradiated over the desired area by a high repetition rate femtosecond fiber laser, under a repetition rate of $1 \mathrm{MHz}$ and two different laser wavelengths (1064 nm and $532 \mathrm{~nm})$, in order to create c-ITO structures. The irradiated substrate was then etched to remove any residual a-ITO film. It was found that high repetition rate of femtosecond fiber laserinduced crystallization significantly improved the surface, crystal, and optical characteristics of a-ITO. The optical transparency increased from 78.8 to $84.9 \%$ at a wavelength of $550 \mathrm{~nm}$. In addition, the periodic-like c-ITO nanostructures with a period of approximately one-quarter of the laser wavelength were observed after irradiation by laser energy near the ablation threshold of the a-ITO thin film.

\section{Acknowledgements}

The authors appreciate the support of the Ministry of the Economic Affairs (MOEA), Taiwan, R.O.C. in conducting this project.

\section{References}

[1] H. Morikawa and M. Fujita, "Crystallization and electrical property changes on the annealing of amorphous indium-oxide and indium-tin-oxide thin films," Thin Solid Films, Vol. 359, 2000.

[2] O. Yavas et al., "Effect of substrate absorption on the efficiency of laser patterning," J. Appl. Phys., Vol. 85, 1999.

[3] A.D. Bonis et al., "Pulsed laser ablation of indium tin oxide in the nano and femtosecond regime: Characterization of transient species," Applied Surface Science, Vol. 252, 2006.

[4] I.B. Sohn et al., "Femtosecond laser patterning of ITO film for display panels," Proc. of the 4th International 
Congress on Laser Advanced Materials Processing, 2006.

[5] M.Y. Xu et al., "F2-laser patterning of indium tin oxide (ITO) thin film on glass substrate," Appl. Phys. A, Vol. 75, 2006.

[6] H.W. Choi et al., "Direct-write patterning of indiumtin-oxide film by high pulse repetition frequency femtosecond laser ablation," Applied Optics, Vol. 46, 2007.

[7] G. RAČIUKAITIS et al., "Patterning of ITO layer on glass with high repetition rate picosecond lasers," Journal of Laser Micro/Nanoengineering, Vol. 2, 2007.

[8] S.J. Kwon "Patterning properties of Indium-Tin Oxide layer depending on the irradiation conditions of the Nd:YVO4 laser beam," Japanese Journal of Applied Physics, Vol. 47, 2008.

[9] G. Legeay, et al., "Excimer laser beam/ITO interaction: from laser processing to surface reaction," physica status solidi (c), Vol. 5, 2008.

[10] R.R. Gattass et al., "Micromachining of bulk glass with bursts of femtosecond laser pulses at variable repetition rates," Opt. Express, Vol. 14, 2006.

[11] S. Juodkazis et al., "Thermal accumulation effects in three-dimensional recordings by picosecond pulses," Appl. Phys. Lett., Vol. 85, 2004.

[12] C.W. Cheng, W.C. Shen, C.Y. Lin, Y.J, Lee, and J.S. Chen, "Fabrication of micro/nano crystalline ITO structures by femtosecond laser pulses," 10th annual Conference on Laser Ablation, Singapore, 2009.

[13]X.C. Wang et al., "Femtosecond pulsed laser-induced periodic surface structures on GaN/sapphire," Applied Surface Science, Vol. 252, 2005.

[14] T.Q. Jia et al., "Formation of nanogratings on the surface of a ZnSe crystal irradiated by femtosecond laser pulses," Phys. Rev. B, Vol. 72, 2005.

[15] B.C. Stuart et al., "Laser-induced damage in dielectrics with nanosecond to subpicosecond pulses," Phys. Rev. Lett., Vol. 74, 1995.

[16] M. Zamfirescu et al., "Femtosecond laser induced periodic surface structure on $\mathrm{ZnO}$ thin film," Journal of Laser Micro/Nanoengineering, Vol. 4, 2009.

(Received: June 7 , 2010, Accepted: October 14, 2010) 